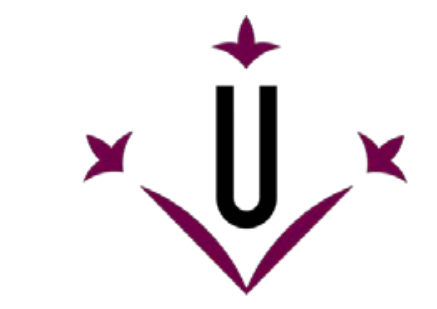

Universitat de Lleida

Document downloaded from:

http://hdl.handle.net/10459.1/66511

The final publication is available at:

https://doi.org/10.1002/jgt.20521

Copyright

(c) Wiley, 2011 


\title{
Vertex-transitive graphs that remain connected after failure of a vertex and its neighbors
}

\author{
Y. O. Hamidoune* $\quad$ A. Lladó ${ }^{\dagger} \quad$ S. C. López ${ }^{\ddagger}$
}

\begin{abstract}
A $d$-regular graph is said to be superconnected if any disconnecting subset with cardinality at most $d$ is formed by the neighbors of some vertex.

A superconnected graph that remains connected after the failure of a vertex and its neighbors will be called vosperian.

Let $\Gamma$ be a vertex-transitive graph of degree $d$ with order at least $d+4$. We give necessary and sufficient conditions for the vosperianity of $\Gamma$. Moreover, assuming that distinct vertices have distinct neighbors, we show that $\Gamma$ is vosperian if and only if it is superconnected.

Let $G$ be a group and let $S \subset G \backslash\{1\}$ with $S=S^{-1}$. We show that the Cayley graph, Cay $(G, S)$, defined on $G$ by $S$ is vosperian if and only if $G \backslash(S \cup\{1\})$ is not a progression and for every non trivial subgroup $H$ and every $a \in G$,
\end{abstract}

$$
|(H \cup H a)(S \cup\{1\})| \geq \min (|G|-1,|H \cup H a|+|S|+1) .
$$

If moreover $S$ is aperiodic, then $\operatorname{Cay}(G, S)$ is vosperian if and only if it is superconnected.

\section{Introduction}

In this section, we give intuitive definitions for some concepts. More formal definitions will be given in Section 2.

An ordered pair $\Gamma=(V, E)$, where $V$ is a set and $E$ is a set of pairs of distinct elements of $V$ will be called a graph. The elements of $V$ will be called vertices and the elements of $E$ edges. The neighbors of a vertex $x$ will be denoted by $\Gamma(x)$. The graph $\Gamma$ will be called $d$-regular if $|\Gamma(x)|=d$, for all $x \in V$. We will restrict ourselves to the case of $d$-regular graphs.

An automorphism of $\Gamma=(V, E)$ is a bijection $f: V \rightarrow V$ such that $f(\Gamma(x))=\Gamma(f(x))$, for every $x \in V$. A graph is said to be vertex-transitive, if for any pair $x, y \in V$, there is an automorphism that maps $x$ into $y$. Let $G$ be a group and let $S \subset G \backslash\{1\}$ with $S=S^{-1}$. The

\footnotetext{
${ }^{*}$ Université Pierre et Marie Curie, E. Combinatoire, Case 189, 4 Place Jussieu, 75005 Paris, France. yha@ccr.jussieu.fr

${ }^{\dagger}$ Universitat Politècnica de Catalunya, Dept. Matemàtica Apl. IV; Jordi Girona, 1, E-08034 Barcelona, Spain. allado@ma4 .upc.edu

${ }^{\ddagger}$ Universitat Politècnica de Catalunya, Dept. Matemàtica Apl. IV; Jordi Girona, 1, E-08034 Barcelona, Spain. susana@ma4.upc.edu
} 
graph $\operatorname{Cay}(G, S)=(G, E)$, where $E=\left\{(x, y): x^{-1} y \in S\right\}$ is called a Cayley graph. Note that the left translation $\gamma_{a}: x \mapsto a x$ is an automorphism of $\operatorname{Cay}(G, S)$.

The graph $\Gamma$ is said to be connected if there is a path connecting two arbitrary vertices. The connectivity of a non complete graph is usually defined as the minimal cardinality of a cutset (a subset of $V$, whose removal produces a graph with more than one connected component).

In order to avoid non-interesting situations, we shall assume from now on that $|V| \geq d+4$.

By a small cutset we shall mean a cutset with cardinality at most $d$. Removing $\Gamma(x)$ of $\Gamma$, $\{x\}$ becomes isolated. The graph $\Gamma$ is said to be superconnected if every small cutset is $\Gamma(x)$ for some $x \in V$. According to our definition, the connectivity of a superconnected graph is necessarily equal to $d$. This notion, introduced by Boesch and Tindell [9], was also investigated by Fiol, Fàbrega and Escudero [16], Fiol [14, 15], Balbuena, Carmona, Fàbrega and Fiol [3, 5], Balbuena and Carmona [2], Marcote, Balbuena and Pelayo [30] and more recently by Balbuena, Marshall and Montejano [7].

If every small cutset creates exactly two connected components one of them with size equal to 1 , the graph $\Gamma$ is said to be vosperian. This notion was used by the authors of [24] as a step in their characterization of superconnected Abelian Cayley digraphs. Note that, a vosperian graph is superconnected. As observed by Meng [31], the notion of a vosperian graph coincide with the notion of a hyperconnected graph, introduced independently by Boesch [8], if $|V| \neq d+3$.

A sharper measure of network vulnerability is given by the notion of extraconnectivity introduced by Fàbrega and Fiol [12]. Extraconnectivity was investigated by Fàbrega and Fiol [13], by Balbuena, Carmona, Fàbrega and Fiol [4], by Balbuena [1], and more recently by Balbuena et al. [6]. Also the concept of $k$-isoperimetric numbers (or $k$-isoperimetric connectivity) introduced by Hamidoune in [19] provides another sharper measure of network vulnerability.

A recursive characterization of vosperian and superconnected Abelian Cayley digraphs where obtained by the authors of [24]. One of the authors obtained a non-recursive characterization using the 2-atoms.

A description of superconnected and vosperian vertex and edge-transitive graphs was given by Meng [31]. Bipartite superconnected and vosperian vertex-transitive graphs were described by Liang, Meng and Zhang [27]. Also superconnected and vosperian vertex-transitive graphs with degree at most 6 were described by Wang and Meng [37] and Tian and Meng [35, 36]. The main tool in the proof of all these results is a method introduced by one of the authors [20].

The paper is organized as follows. In Section 2, we introduce the isoperimetric connectivity. In Section 3, we give a necessary and sufficient condition for a vertex-transitive graph to be vosperian in terms of the automorphism group.

Let $G$ be a group. Let $S$ be a subset of $G$ with $S=S^{-1}$ and let $H$ be a subgroup of $G$. The Sabidussi quotient is the graph $\mathcal{S}(G, H, S)$ defined on the set of left $H$-cosets such that $x H y H$ is an edge in $\mathcal{S}(G, H, S)$ if and only if $y H=x s H$ for some $s \in S$. Sabidussi [33] proved that every vertex transitive graph is a Sabidussi quotient. The Sabidussi quotient is a Cayley graph if $H$ is a normal subgroup, but in general, it is not a Cayley graph. In Section 4, we construct a vertex-transitive graph $\bar{\Gamma}$ by collapsing twins (pair of vertices that have the same neighbors) from a given vertex-transitive graph $\Gamma$. We show that $\Gamma$ is superconnected if and 
only if $\bar{\Gamma}$ is superconnected. Due to that we can restrict ourselves to twin free graphs. Let $Q=\{x: x S=S\}$ and let $\Lambda$ be the Cayley graph defined on $G$ by $S$. The quotient graph $\bar{\Lambda}$ is just the Sabidussi quotient $\mathcal{S}(G, Q, S)$.

In Section 5, we show that a superconnected vertex-transitive graph remains connected after deleting a vertex and its neighbors, whenever distinct vertices have distinct neighbors. As a corollary, we show that any vertex-transitive graph $\Gamma=(V, E)$ of degree $d$ coprime to $|V|$, is superconnected if and only it is vosperian. It follows that there is only a very small number of superconnected vertex-transitive graphs that are non-vosperian. In Section 6, we investigate the case of Cayley graphs:

Let $S$ be a generating subset of a finite group $G$, with $1 \notin S, S=S^{-1}$ and $|G|-4 \geq|S|$. Put $\tilde{S}=S \cup\{1\}$. We prove that $\Gamma=\operatorname{Cay}(G, S)$ is vosperian if and only if the following hold:

(i) $G \backslash \tilde{S}$ is not a progression.

(ii) For every non trivial subgroup $H$ and every $a \in G$,

$$
|(H \cup H a) \tilde{S}| \geq \min (|G|-1,|H \cup H a|+|S|+1) .
$$

This characterization of vosperian Cayley graphs extends the obtained in the Abelian case by one of the authors in [20]. As a corollary, we prove the following:

If $S$ is aperiodic, then $\operatorname{Cay}(G, S)$ is superconnected if and only if $G \backslash \tilde{S}$ is not a progression, and for every non trivial subgroup $H$ and every $a \in G$,

$$
|(H \cup H a) \tilde{S}| \geq \min (|G|-1,|H \cup H a|+|S|+1) .
$$

\section{Terminology and preliminaries}

Let $G$ be a group and let $S$ be a subset of $G$. The subgroup generated by $S$ will be denoted by $\langle S\rangle$. Let $A, B$ be subsets of $G$. The Minkowski product is defined as

$$
A B=\{x y: x \in A \text { and } y \in B\} .
$$

Let $\Gamma=(V, E)$ be a graph. The minimal degree is $\delta_{\Gamma}=\min \{|\Gamma(x)|: x \in V\}$. For a subset $X \subset V(G)$, we write

$$
\Gamma(X)=\bigcup_{x \in X} \Gamma(x)
$$

A pair of vertices $\{x, y\}$ is said to be a twin pair if $\Gamma(x)=\Gamma(y)$, a friend pair if, $x \in \Gamma(y)$ and $|\Gamma(x) \cap \Gamma(y)| \geq|\Gamma(x)|-2=|\Gamma(y)|-2$. Figure 1 shows two friend pair and a twin pair.

Given a subset $X \subset V$, the boundary of $X$ is the set $\partial_{\Gamma}(X)=\Gamma(X) \backslash X$, and the exterior of $X$ is $\nabla_{\Gamma}(X)=V \backslash(X \cup \Gamma(X))$. If the context is clear the reference to $\Gamma$ will be omitted. For every set $X$, we have a partition (with possibly empty parts) $V=X \cup \partial(X) \cup \nabla(X)$. Notice 

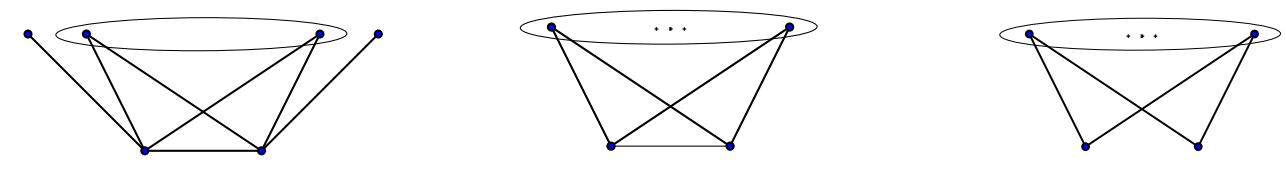

Figure 1: Two friend pairs and a twin pair.

that $\Gamma(X) \cap \nabla(X)=\emptyset$. The graph $\Gamma$ is $k$-separable if there is a subset $X \subset V$ such that $\min \{|X|,|\nabla(X)|\} \geq k$. If $\Gamma$ is $k$-separable, its $k$ th-isoperimetric connectivity is

$$
\kappa_{k}(\Gamma)=\min \{|\partial(X)|: X \subset V, \min \{|X|,|\nabla(X)|\} \geq k\} .
$$

A set $F \subset V$ is called a $k$-fragment if $\min \{|F|,|\nabla(F)|\} \geq k$ and $|\partial(F)|=\kappa_{k}(\Gamma)$. A $k$-atom is a $k$-fragment of minimum cardinality.

Notice that $\kappa_{1}$ is precisely the connectivity of the graph as defined in Graph Theory text books like $[10,11]$. So we shall write $\kappa$ instead of $\kappa_{1}$. Also the 1-atom coincide with the notion of atom introduced independently by Mader [28] and Watkins [38].

The notions $k$-isoperimetric connectivity and $k$-atoms introduced by Hamidoune in [19] gives a formalism to go more deeply than the traditional connectivity. Applications of these concepts may be found in [19, 21, 22, 23] and in the survey paper by Serra [34]. The corresponding notion for edge connectivity was independently investigated by van den Heuvel and Jackson [26] and by the authors of [25].

We recall the following duality lemma:

Lemma A ([23]) Let $\Gamma=(V, E)$ be a finite $k$-separable graph. If $X$ is a $k$-fragment then

$$
\partial(\nabla(X))=\partial(X) \quad \text { and } \quad \nabla(\nabla(X))=X .
$$

In particular, $\nabla(X)$ is a $k$-fragment.

The following intersection property is helpful:

Theorem B ([19]) Let $\Gamma=(V, E)$ be a finite $k$-separable graph. Let $A$ be a $k$-atom and let $F$ be a k-fragment.

(i) If $|A \cap F| \geq k$ then $A \subset F$. In particular,

(ii) two distinct $k$-atoms intersect in at most $k-1$ elements.

For $k=1$, (i) produces a result of Mader [29] and (ii) produces a result proved independently by Mader [28] and Watkins [38].

We note that Theorem B above is originally formulated for directed graphs with loops in [19]. Clearly the addition (of deletion) of loops does not modify the notions of $k$-fragments 
and $k$-isoperimetric connectivity. Working with loops lead to more concise formulaes since $\Gamma(A)=A \cup \Gamma(A)$ in this case.

Again the notion of a super-atom used in $[17,18]$ is a special case of the notion of a 2-atom introduced in [19]. The present formulation used in $[19,20]$ is more suitable for applications. A minimum cutset $T$ is a cutset $T$ with $|T|=\kappa$. Notice that every minimum cutset is $T=\partial(F)$, where $F$ is 1 -fragment. The graph $\Gamma$ is said to be vosperian if either $\Gamma$ is not 2 -separable or $\kappa_{2}(\Gamma) \geq \delta+1$. The graph $\Gamma$ is said to be superconnected if every minimum cutset is of the form $\Gamma(x)$. Recall the following result:

Theorem C ([18]) Let $G$ be a finite group and $S \subset G \backslash\{1\}$ with $S=S^{-1}$. If $H$ is a 1-atom of $\operatorname{Cay}(G, S)$ containing 1 , then $H$ is a subgroup.

As a main tool, we use the following result proved by one of the authors in connection with Additive Number Theory in [21] and generalized to the infinite case in [23, Theorem 9.3.].

Theorem D ([21]) Let $\Gamma=(V, E)$ be a finite 2-separable vertex-transitive graph of degree $d$. Let $A$ be a 2-atom. If $|A| \geq 3$ then one of the following holds:

(i) Every vertex of $V$ is contained in at most two distinct 2-atoms.

(ii) $|A| \leq \kappa_{2}(\Gamma)-d+2$.

Notice that this result is also a basic tool in the work of Meng [31] and Meng and Zhang [32]. We recall here some simple preliminary lemmas. We begin with some equivalent assertions that can be taken as a definition of a non-vosperian graph.

Lemma $\mathbf{E}$ (folklore) Let $\Gamma=(V, E)$ be a finite $d$-regular graph with $|V| \neq d+3$. The following conditions are equivalent:

(i) $\Gamma$ is not 2 -separable or $\kappa_{2}(\Gamma) \geq d+1$.

(ii) A 1-fragment of $\Gamma$ has the form $\{x\}$ or $V \backslash(\{x\} \cup \Gamma(x))$.

(iii) A 1-fragment of $\Gamma$ has size equal to 1 or $|V|-d-1$.

(iv) Every minimum cutset isolates a vertex and, $V \backslash(\{x\} \cup \Gamma(x))$ is connected for every $x \in V$.

Proof. Suppose that (i) holds and let $T$ be a minimum cutset. Hence $T=\partial(F)$, where $F$ is a 1-fragment. Since $|\partial(F)|=d$, we have $\min \{|F|,|\nabla(F)|\}=1$. Hence either $F=\{x\}$ or $\nabla(F)=\{x\}$. In the second case, by Lemma A, we have $F=\nabla(\nabla(F))=V \backslash(\Gamma(x) \cup\{x\})$.

Obviously, (ii) implies (iii). Now, suppose that (iii) holds and let $\partial(F)$ be a minimum cutset. If $F$ is a 1-fragment of cardinality $|V|-d-1$, then $|\nabla(F)|=1$, and by Lemma A, $\partial(\nabla(F))=\partial(F)$. Moreover, let $C_{1}, \ldots, C_{k}$ be the connected components of $V \backslash(\{x\} \cup \Gamma(x))$, 
where $x$ is a vertex. If $k>1$ then $C_{1} \cup\{x\}$ is a 1-fragment with size greater than 1 . It follows that $\left|V\left(C_{1}\right)\right|+1=|V|-d-1$ which implies $k=2$ and $\left|V\left(C_{2}\right)\right|=1$. A symmetric argument shows that $\left|V\left(C_{1}\right)\right|=1$ as well so that $|V|=d+3$, a contradiction. Hence $k=1$ and (iv) holds.

Finally, suppose that (iv) holds and that $\Gamma$ is 2 -separable with $\kappa_{2}(\Gamma)=d$. Take a set $X$ with $\min \{|X|,|\nabla(X)|\} \geq 2$ and $|\partial(X)|=d$. It follows by (iv) that $\partial(X)$ isolates a vertex $a$, so that $\Gamma(a)=\partial(X)$. Now, $V \backslash(\{a\} \cup \Gamma(a))$ has two connected components contradicting (iv). So, (iv) implies (i).

Lemma $\mathbf{F}$ (folklore) Let $\Gamma$ be a finite d-regular graph with $\kappa(\Gamma)=d$. The following conditions are equivalent:

(i) $\Gamma$ is superconnected.

(ii) For every 1-fragment $F$ of $\Gamma, V \backslash \partial(F)$ has an isolated vertex.

Proof. Trivially, (i) implies (ii). Moreover, suppose that $T$ is a cutset. There is a 1-fragment $F$ such that $\partial(F)=T$. Take an isolated vertex $x$ of $V \backslash T$. Clearly $T \supset \Gamma(x)$. Since $\kappa(\Gamma)=d$, we have $T=\Gamma(x)$.

Lemma $\mathbf{G}$ (folklore) Let $T$ be a minimum cutset, with $|T|=d$ and let $C$ be a union of connected components of $V \backslash T$. If $|V(C)| \geq 2$ and $|V \backslash V(C)| \geq d+2$, then $V(C)$ is a 2-fragment.

\section{$3 \quad$ Vosperian vertex-transitive graphs}

Let $\Gamma=(V, E)$ be a vertex-transitive graph and let $\Phi$ be a set of automorphisms of $\Gamma$. For a pair $X, Y \subset V$, we shall write $\Phi_{X, Y}=\{f \in \Phi: f(X) \subset Y\}$. The following result encodes in terms of the automorphism group, a necessary and sufficient condition for a vertex-transitive graph to be non-vosperian:

Theorem 1 Let $\Gamma=(V, E)$ be a finite vertex-transitive graph of degree $d$ with $d \leq|V|-4$. Let $\mathcal{A}$ be a subgroup of the automorphism group which is transitive on $V$ and let $v \in V$. Then $\Gamma$ is non-vosperian if and only if one of the following conditions holds:

(i) There exists a friend pair.

(ii) There exists a twin pair.

(iii) There are an $\alpha \in \mathcal{A}$ and a set $M$, with $|M| \geq 2,|M \cup \partial(M)| \leq|V|-2$ and $|\partial(M)| \leq d$, such that $\mathcal{A}_{v, M}=\mathcal{A}_{M, M} \cup \mathcal{A}_{M, M} \alpha$. 
Proof. Let $\{x, y\}$ be a twin pair. We have $|\Gamma(\{x, y\})|=2 d-|\Gamma(x) \cap \Gamma(y)|=2 d-d=d$. Thus $|\partial(\{x, y\})|=|\Gamma(\{x, y\})|=d$. Let $\{x, y\}$ be a friend pair. We have $|\Gamma(\{x, y\})|=2 d-$ $|\Gamma(x) \cap \Gamma(y)| \leq 2 d-(d-2)=d+2$. Thus $|\partial(\{x, y\})|=|\Gamma(\{x, y\})|-2 \leq d$. Thus, any of the three conditions implies that $\Gamma$ is 2 -separable and $\kappa_{2}(\Gamma) \leq d$, and hence $\Gamma$ is non-vosperian, by Lemma E.

Let us now prove the necessity. If $\Gamma$ is non-vosperian, then $\Gamma$ is 2 -separable and $\kappa_{2}(\Gamma) \leq d$. Let $M$ be a 2-atom of $\Gamma$, in particular $|M| \geq 2,|M \cup \partial(M)| \leq|V|-2$ and $|\partial(M)| \leq d$. We may assume that $|M| \geq 3$, otherwise (i) or (ii) holds. Put $\mathcal{A}_{v}=\mathcal{A}_{v, M}$ and $\mathcal{A}_{M}=\mathcal{A}_{M, M}$.

If $\mathcal{A}_{v}=\mathcal{A}_{M}$ then (iii) holds with $\alpha$ the identity element. Otherwise choose an $\alpha \in \mathcal{A}_{v} \backslash \mathcal{A}_{M}$, that is $\alpha(M) \neq M$ and we have that $\alpha^{-1}(M)$ is a 2-atom different from $M$. Since $|M| \geq 3$, Theorem $\mathrm{D}$ implies that there are precisely two 2-atoms containing $v$. Therefore, for each $f \in \mathcal{A}_{v} \backslash \mathcal{A}_{M}$ we have $f^{-1}(M)=\alpha^{-1}(M)$. It follows that $f \alpha^{-1}(M)=M$ so that $f \alpha^{-1} \in \mathcal{A}_{M}$.

\section{The twin reduction}

In this section, we construct a vertex transitive graph $\bar{\Gamma}$ by collapsing twins from a given vertextransitive graph $\Gamma$ and we show that $\Gamma$ is superconnected if and only if $\bar{\Gamma}$ is superconnected. Due to that we can restrict ourselves to twin free graphs.

Let $\mathcal{R}$ be an equivalence relation on a set $V$ and let $\pi: V \rightarrow V / \mathcal{R}$ be the canonical epimorphism. A subset $S \subset V$ is said to be saturated if $S=\pi^{-1}(\pi(S))$ (or, equivalently, if $S$ is a union of equivalence classes).

Let $\Gamma=(V, E)$ and let $x, y \in V$. The relation $\Gamma(x)=\Gamma(y)$ is clearly an equivalence relation that will be denoted by $x \sim_{\Gamma} y$. The twin class of a vertex $a$ is $\pi^{-1}(\pi(a))=\left\{x: x \sim_{\Gamma} a\right\}$, since it contains all vertices that have the same neighbors of $a$. Since $\pi$ is surjective, $\pi \circ \pi^{-1}=\mathrm{id}_{V / \sim_{\Gamma}}$.

Lemma 2 Let $\Gamma=(V, E)$ be a graph. If $S$ is a saturated subset of $V$ then $\partial_{\Gamma}(S)$ is saturated.

Proof. Assume that $x \in \partial_{\Gamma}(S)$ and that $y \sim_{\Gamma} x$. Hence there exists a $b \in S$ such that $x \in \Gamma(b)$. Since $\Gamma(x)=\Gamma(y)$, we have $y \in \Gamma(b)$. Thus $y \notin \nabla_{\Gamma}(S)$. Since $S$ is saturated, we have $y \notin S$, otherwise $x \in S$, a contradiction. It follows that $y \in \partial_{\Gamma}(S)$.

The quotient graph is by definition $\bar{\Gamma}=\left(V / \sim_{\Gamma}, \mathcal{E}\right)$, where

$$
\mathcal{E}=\{\{X, Y\}: \text { There are } x \in X \text { and } y \in Y \text { such that }\{x, y\} \in E\} .
$$

Lemma 3 Let $\Gamma=(V, E)$ be a finite graph. Then $\pi: V \rightarrow V / \sim_{\Gamma}$ is a graph homomorphism. Moreover, if $X \subset V$ and $Y \subset V / \sim_{\Gamma}$, then

$$
\begin{array}{r}
\pi(\Gamma(X))=\bar{\Gamma}(\pi(X)), \\
\Gamma\left(\pi^{-1}(Y)\right)=\pi^{-1}(\bar{\Gamma}(Y)) .
\end{array}
$$


Proof. Clearly, $\pi$ is a graph homomorphism, since $\{\pi(x), \pi(y)\} \in \mathcal{E}$ if and only if for all $x^{\prime} \in \pi^{-1}(\pi(x))$ and $y^{\prime} \in \pi^{-1}(\pi(y)),\left\{x^{\prime}, y^{\prime}\right\} \in E$. Hence, for all $x \in X, \pi(\Gamma(x))=\bar{\Gamma}(\pi(x))$, and equality (1) follows. Take $y \in V / \sim_{\Gamma}$, and $z \in \pi^{-1}(\bar{\Gamma}(y))$. Choose $c \in V$ such that $\pi(c)=$ $y$. Since $\pi(z) \in \bar{\Gamma}(\pi(c))$ implies $z \in \Gamma(c)$, we have that $z \in \Gamma\left(\pi^{-1}(y)\right)$. Thus, $\pi^{-1}(\bar{\Gamma}(y)) \subset$ $\Gamma\left(\pi^{-1}(y)\right)$. Take now $x \in \Gamma\left(\pi^{-1}(y)\right)$. Take $a$ with $\pi(a)=y$ such that $x \in \Gamma(a)$. It follows that $\pi(x) \in \pi(\Gamma(a))=\bar{\Gamma}(y)$. Thus $x \in \pi^{-1}(\bar{\Gamma}(y))$. Hence, $\Gamma\left(\pi^{-1}(y)\right) \subset \pi^{-1}(\bar{\Gamma}(y))$. Therefore, $\Gamma\left(\pi^{-1}(y)\right)=\pi^{-1}(\bar{\Gamma}(y))$. It follows that

$$
\pi^{-1}(\bar{\Gamma}(Y))=\bigcup_{y \in Y} \pi^{-1}(\bar{\Gamma}(y))=\bigcup_{y \in Y} \Gamma\left(\pi^{-1}(y)\right)=\Gamma\left(\pi^{-1}(Y)\right)
$$

proving (2).

Lemma 4 Let $\Gamma=(V, E)$ be a finite graph. Let $f$ be an automorphism of $\Gamma$. Then, for all $x \in V$

$$
f\left(\pi^{-1}(\pi(x))\right)=\pi^{-1}(\pi(f(x)))
$$

Moreover, there exists an unique automorphism $\bar{f}$ of $\bar{\Gamma}$ such that $\bar{f} \circ \pi=\pi \circ f$.

Proof. Let $f$ be an automorphism of $\Gamma$. Let us show first, that $x \sim_{\Gamma} y$ holds if and only if $f(x) \sim_{\Gamma} f(y)$. In particular $f\left(\pi^{-1}(\pi(x))\right)=\pi^{-1}(\pi(f(x)))$

Assume that $x \sim_{\Gamma} y$. Then $\Gamma(x)=\Gamma(y)$. By the definition of an automorphism, we have $\Gamma(f(x))=f(\Gamma(x))=f(\Gamma(y))=\Gamma(f(y))$. The other implication follows by considering $f^{-1}$. Thus, $\bar{f}(\pi(x))=\pi(f(x))$ defines an injective map on $V / \sim_{\Gamma}$. Let us show that $\bar{f}$ is an homomorphism of $\bar{\Gamma}$.

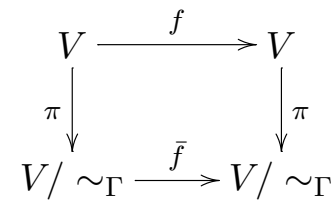

Assume that $\pi(x) \in \bar{\Gamma}(\pi(y))$. By the above observation, this happens if and only if $x \in \Gamma(y)$. Since $f$ is an automorphism, $f(x) \in f(\Gamma(y))=\Gamma(f(y))$. Thus, $\pi(f(x)) \in \bar{\Gamma}(\pi(f(y)))$, and hence, $\bar{f}(\pi(x)) \in \bar{\Gamma}(\bar{f}(\pi(y)))$. Therefore, $\bar{f}$ is an automorphism.

Lemma 5 Let $\Gamma=(V, E)$ be a vertex-transitive graph of degree $d$ and $v \in V$. Let $\tau=$ $\left|\pi^{-1}(\pi(v))\right|$. Then $\bar{\Gamma}$ is a vertex transitive graph with degree $d / \tau$ without twins.

Proof. Let $v, w \in V$ and let $f$ be an automorphism with $f(v)=w$. By Lemma 4 , there exists an automorphism $\bar{f}$ of $\bar{\Gamma}$ such that $\bar{f} \circ \pi=\pi \circ f$. Thus, $\bar{f}(\pi(v))=\pi(f(v))=\pi(w)$. Hence, $\bar{\Gamma}$ is a vertex transitive graph. By (3), we have $\left|\pi^{-1}(\pi(x))\right|=\left|\pi^{-1}(\pi(v))\right|$, for all $x$. In particular,

$$
\tau|V / \sim \Gamma|=|V|
$$

Since $\Gamma(v)=\partial_{\Gamma}\left(\pi^{-1}(\pi(v))\right.$, by Lemma $2 \Gamma(v)$ is saturated. Therefore, $\tau|\bar{\Gamma}(\pi(v))|=|\Gamma(v)|=d$. 
Let us prove now that $\bar{\Gamma}$ has no twins. Note that $\pi(z) \in \bar{\Gamma}(\pi(x))$ implies that, $\pi^{-1}(\pi(z)) \subset$ $\Gamma(x)$. Hence, if $\pi(x) \sim_{\Gamma} \pi(y)$ then $\Gamma(x)=\Gamma(y)$, and therefore, $\pi(x)=\pi(y)$.

By the previous proof, we also conclude the following:

Corollary 6 Let $\Gamma=(V, E)$ be a vertex-transitive graph of degree $d, v \in V$. Let $\tau$ be the cardinality of a twin class. Then, $|V|=\tau|V / \sim \Gamma|$, and $d=\tau|\bar{\Gamma}(\pi(v))|$.

In particular, if $\operatorname{gcd}(|V|, d)=1$ then $\tau=1$.

Lemma 7 Let $\Gamma$ be a vertex-transitive graph of degree $d$ with $\kappa(\Gamma)=d$. Let $\tau$ be the cardinality of a twin class. Then $\kappa(\bar{\Gamma})=d / \tau$. Moreover,

(i) If $F$ is a 1-fragment of $\bar{\Gamma}$ then, $\pi^{-1}(F)$ is a saturated 1-fragment of $\Gamma$, and

$$
\partial_{\Gamma}\left(\pi^{-1}(F)\right)=\pi^{-1}\left(\partial_{\bar{\Gamma}}(F) .\right.
$$

(ii) If $X$ is a saturated 1-fragment of $\Gamma$ then, $\pi(X)$ is a 1-fragment of $\bar{\Gamma}$.

Proof. Let $F$ be a 1-fragment of $\bar{\Gamma}$. Let us show first that, $\pi^{-1}(F) \cup \Gamma\left(\pi^{-1}(F)\right) \neq V$. By equality (2), we have $\pi\left(\pi^{-1}(F) \cup \Gamma\left(\pi^{-1}(F)\right)\right)=F \cup \bar{\Gamma}\left(\pi\left(\pi^{-1}(F)\right)\right)=F \cup \bar{\Gamma}(F)$. Hence, since $F \cup \bar{\Gamma}(F) \neq V / \sim$, we have $\pi^{-1}(F) \cup \Gamma\left(\pi^{-1}(F)\right) \neq V$. By the (isoperimetric) definition of $\kappa$ we have, $\left|\Gamma\left(\pi^{-1}(F)\right) \cup \pi^{-1}(F)\right| \geq\left|\pi^{-1}(F)\right|+d$. Since $\left|\Gamma\left(\pi^{-1}(F)\right) \cup \pi^{-1}(F)\right|=|F \cup \bar{\Gamma}(F)| \tau$, it follows that $|F \cup \bar{\Gamma}(F)| \geq|F|+d / \tau$. By Lemma 5 , we have that $d / \tau$ is the degree of $\bar{\Gamma}$, in particular, $\kappa(\bar{\Gamma}) \leq d / \tau$, which implies,

$$
|F \cup \bar{\Gamma}(F)| \leq|F|+d / \tau .
$$

Thus, $\kappa(\bar{\Gamma})=d / \tau$. In particular, $\pi^{-1}(F)$ is a (saturated) 1-fragment of $F$. Moreover, by equality (2),

$$
\partial_{\Gamma}\left(\pi^{-1}(F)\right)=\pi^{-1}(\bar{\Gamma}(F)) \backslash \pi^{-1}(F) \subset \pi^{-1}(\bar{\Gamma}(F) \backslash F) .
$$

It follows that, $\left|\partial_{\Gamma}\left(\pi^{-1}(F)\right)\right|=\left|\pi^{-1}(\bar{\Gamma}(F) \backslash F)\right|$, which implies, $\partial_{\Gamma}\left(\pi^{-1}(F)\right)=\pi^{-1}\left(\partial_{\bar{\Gamma}}(F)\right.$, and (i) holds.

Now, let $X$ be a saturated 1-fragment of $F$. By definition, $|X \cup \Gamma(X)|=|X|+d$, and $\partial(X)$ is saturated by Lemma 2 .

Hence, and by equality (1), we have

$$
|\pi(X) \cup \pi(\Gamma(X))|=\frac{|X|}{\tau}+\frac{d}{\tau} .
$$

Therefore, $\pi(X)$ is a 1-fragment, and the result follows.

Theorem 8 Let $\Gamma$ be a vertex-transitive graph with $\kappa(\Gamma)=d$. Then, $\bar{\Gamma}$ is superconnected if and only if $\Gamma$ is superconnected. 
Proof. Suppose that $\Gamma$ is superconnected. Take a 1-fragment $F$ of $\bar{\Gamma}$. By the above Lemma $\pi^{-1}(F)$ is a 1-fragment of $\Gamma$. There is $v \in V$, such that $\Gamma(v)=\partial_{\Gamma}\left(\pi^{-1}(F)\right)$. It follows that, $\pi(\Gamma(v))=\pi\left(\partial_{\Gamma}\left(\pi^{-1}(F)\right)\right)$ and hence by Lemma 7 and equality $(1), \bar{\Gamma}(\pi(v))=\partial_{\bar{\Gamma}}(F)$. Therefore, $\bar{\Gamma}$ is superconnected.

Now, suppose that $\bar{\Gamma}$ is superconnected. Let $X$ be a 1 -fragment of $\Gamma$. We may assume that $X$ contains no isolated vertex $u$, otherwise, $\partial_{\Gamma}(X)=\Gamma(u)$. We shall prove now that $X$ is saturated. Take $x \in X$, and $y \sim_{\Gamma} x$. Since $x$ is not isolated, there is $a \in X$ such that $a \in \Gamma(x)$. Thus $y \in \Gamma(a)$. Suppose that $y \in \partial_{\Gamma}(X)$. Since $\partial_{\Gamma}(X)$ is a minimum cutset there is $z \in \nabla_{\Gamma}(X) \cap \Gamma(y)$. Since, $x$ and $y$ are twins, it follows that $z \in \Gamma(x)$, a contradiction. Since $X$ is saturated, by Lemma $7, \pi(X)$ is a 1-fragment of $\bar{\Gamma}$. Thus there is a $v \in V$ such that $\bar{\Gamma}(\pi(v))=\partial_{\bar{\Gamma}}(\pi(X))$. By equality (1), it follows that $\pi(\Gamma(v))=\partial_{\bar{\Gamma}}(\pi(X))$, which implies $\pi^{-1}\left(\pi(\Gamma(v))=\pi^{-1}\left(\partial_{\bar{\Gamma}}(\pi(X))\right)\right.$. Hence, by Lemma $2, \Gamma(v)=\partial_{\Gamma}(X)$. Therefore, $\Gamma$ is superconnected.

Notice that some special cases of this result are contained in the papers $[27,31]$.

\section{$5 \quad$ Superconnected vertex-transitive graphs}

The next theorem shows that, for vertex transitive graphs with no twin pairs, the notions of superconnectivity and vosperianity are equivalent.

Theorem 9 Let $\Gamma=(V, E)$ be a vertex-transitive graph of degree $d$ without a twin pair. If every small cutset consists of some vertex neighbors, then for every $x \in V, V \backslash(\{x\} \cup \Gamma(x))$ is connected.

That is, $\Gamma$ is vosperian if and only if it is superconnected.

Proof. Suppose the contrary and let $C_{1}, C_{2}$ be two distinct connected components of $V \backslash(\{x\} \cup$ $\Gamma(x))$. Since $C_{i}$ is a connected component of $V \backslash(\{x\} \cup \Gamma(x))$, we have necessarily $\partial\left(C_{i}\right) \subset \Gamma(x)$. Thus, $\kappa(\Gamma)=d$, implies $\partial\left(C_{i}\right)=\Gamma(x)$. For $1 \leq i \leq 2$, we have $\left|C_{i}\right| \geq 2$. Otherwise, $C_{i}=\{z\}$ for some $z$ and $\{x, z\}$ is a twin pair, a contradiction. Thus, $\Gamma$ is 2 -separable. Let $A$ be a 2 -atom of $\Gamma$ and let $T=\partial(A)$.

Assume first $|A|=2$. In particular, $|\nabla(A)| \geq 3$. Let $A=\{a, b\}$. We must have $b \in \Gamma(a)$, otherwise $\Gamma(a) \cup \Gamma(b) \subset T$. Since $d=|\Gamma(a)|=|\Gamma(b)|=|T|,\{a, b\}$ is a twin pair, a contradiction. Let $T_{0}=\Gamma(a) \cap \Gamma(b)$. Since $d+2=|\Gamma(a) \cup \Gamma(b)|=2 d-|\Gamma(a) \cap \Gamma(b)|$, we have $\left|T_{0}\right|=d-2$. In particular there are distinct vertices $a^{\prime}, b^{\prime}$ such that $\Gamma(a)=\left\{b, a^{\prime}\right\} \cup T_{0}$, and $\Gamma(b)=\left\{a, b^{\prime}\right\} \cup T_{0}$.

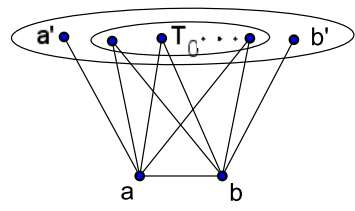

Since every small cutset of $\Gamma$ consists of some vertex neighbors, there exists $u \in V$ such that $\Gamma(u)=T$. Clearly, $\Gamma(a) \neq T$ and $\Gamma(b) \neq T$, and hence $u \in \nabla(A)$. Take an automorphism 
$f$ of $\Gamma$ such that $f(a)=u$. Let $v=f(b)$ and $A^{\prime}=f(A)$. Clearly, $A^{\prime}$ is a 2-atom of $\Gamma$ that contains $u$. Since $f$ is an automorphism, we have $v \in \Gamma(u)=T$. We have $v \notin T_{0}$. Otherwise $\Gamma(\{u, v\}) \supset\{u, v\} \cup \Gamma(u) \cup \Gamma(v) \supset\{u, v\} \cup T \cup\{a, b\}$, and thus $\left|\partial\left(A^{\prime}\right)\right| \geq d+1$, a contradiction. Hence, $v \in\left\{a^{\prime}, b^{\prime}\right\}$. Without loss of generality, we can suppose that $v=a^{\prime}$.

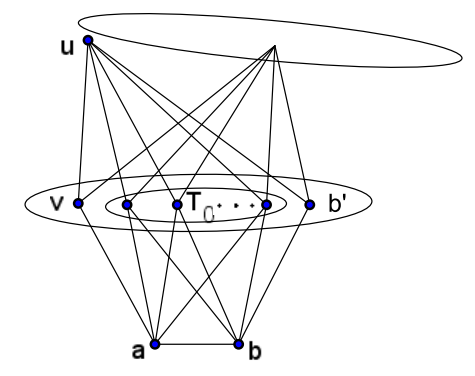

Since by Lemma G, $\nabla(A) \backslash\{u\}$ is a 2 -fragment, $\Gamma(v) \cap(\nabla(A \backslash\{u\})) \neq \emptyset$. It follows that

$$
\left|\partial\left(A^{\prime}\right)\right|>\left|T_{0} \cup\left\{a, b^{\prime}\right\}\right|
$$

a contradiction.

Assume now $|A| \geq 3$. For every $v \in A, \Gamma(v) \neq \partial(A)$, otherwise $\partial(A \backslash\{v\}) \subset \partial(A)$, a contradiction. Since every small cutset of $\Gamma$ consists of some vertex neighbors, there exists $u \in V$ with $\Gamma(u)=\partial(A)$, in particular, $u \in \nabla(A)$. Thus, by Lemma G, $\nabla(A) \backslash\{u\}$ and $A \cup\{u\}$ are 2-fragments. Take $a \in A$ and an automorphism $f$ of $\Gamma$ such that $f(a)=u$. Let $A^{\prime}=f(A)$. Clearly $A^{\prime}$ is a 2-atom of $\Gamma$ that contains $u$. Observe that $a \in \Gamma(b)$, for some $b \in A$, since otherwise $\{a, u\}$ is a twin pair. Now $f(b) \in A^{\prime} \cap \Gamma(u) \subset \partial(A)$. In particular $A^{\prime} \not \subset \nabla(A)$. By Theorem B, $A^{\prime} \cap(\nabla(A) \backslash\{u\})=\emptyset$. We have $A^{\prime} \cap A=\emptyset$. Otherwise $\left|A^{\prime} \cap(A \cup\{u\})\right| \geq 2$, and by Theorem B, $A^{\prime} \subset A \cup\{u\}$, a contradiction. It follows that $X=A^{\prime} \backslash u \subset \partial(A)$. Let $A_{1}=A \backslash \partial(X)$.
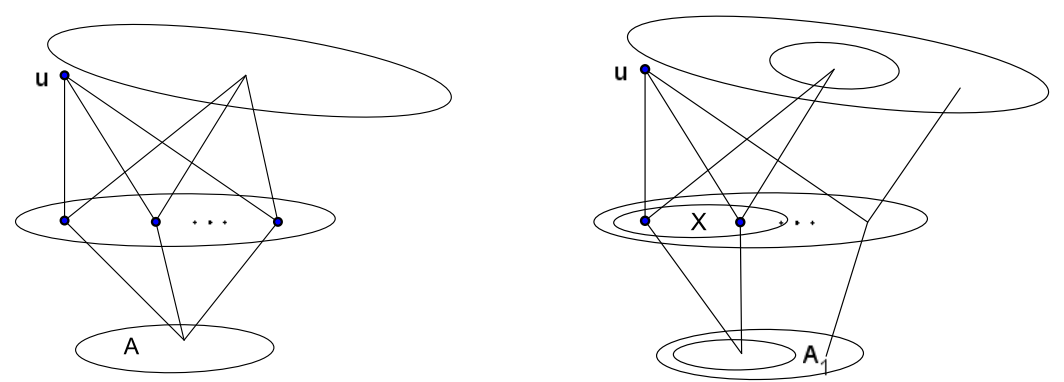

We have $|\partial(X) \cap A| \geq|X|$. Otherwise

$$
\left|\partial\left(A_{1}\right)\right| \leq|X|-1+(|\partial(A)|-|X|)=|\partial(A)|-1=d-1,
$$

contradicting our assumption that $\kappa(\Gamma)=d$. Similarly, $|\partial(X) \cap(\nabla(A) \backslash\{u\})| \geq|X|$. Hence,

$$
\left|\partial\left(A^{\prime}\right)\right|=|\Gamma(u) \backslash X|+|\partial(X) \cap A|+|\partial(X) \cap(\nabla(A) \backslash\{u\})| \geq|\partial(A)|+|X|,
$$

a contradiction proving the first statement of the theorem. 
As we mention before, $\Gamma$ is superconnected if $\Gamma$ is vosperian. By the first part of the theorem, if every small cutset consists of some vertex neighbors, then $V \backslash(\{x\} \cup \Gamma(x))$, is connected for every $x$. Hence, by Lemma $\mathrm{E}, \Gamma$ is vosperian.

Corollary 10 Let $\Gamma$ be a vertex-transitive graph of degree $d \leq|V|-4$, such that $\operatorname{gcd}(|V|, d)=1$. Then, $\Gamma$ is vosperian if and only if it is superconnected.

Proof. By Corollary $6, \tau=1$. The result follows now by Theorem 9 .

\section{Cayley graphs}

In this section, we apply our characterization to the case of Cayley graphs. Then, Theorem 1 yields the following result.

Proposition 11 Let $S$ be a generating subset of a finite group $G$ with $1 \notin S$ and $S=S^{-1}$, such that $\operatorname{Cay}(G, S)$ is non-vosperian. Let $A$ be a 2 -atom of $C a y(G, S)$ with $1 \in A$. Then there are a subgroup $H$ and an element $a \in G$ such that $A=H \cup H a$.

Proof. The result follows easily by Theorem 1 , taking as $\mathcal{A}$ the set of left translations. We give below a direct proof using Theorem $\mathrm{D}$.

We may assume that $|A| \geq 3$, otherwise we may take $H=\{1\}$. Let $H=\{x \in G \mid x A=A\}$. The result clearly holds if $A=H$. Take $a \in A \backslash H$. We shall show that $A=H \cup H a$. Observe that $A$ and $a^{-1} A$ are two distinct 2-atoms containing 1 . Thus by Theorem $\mathrm{D}$, for every $x \in A$, we have $x^{-1} A=A$ or $x^{-1} A=a^{-1} A$. Therefore, either $x \in H$ or $x a^{-1} \in H$. In particular, $A \subset H \cup H a$. But $H A \subset A$, by the definition of $H$.

If $G$ is Abelian, then $A$ is a subgroup [20]. The following example shows that in the nonabelian case, this is not necessarily true.

Example. Let $G$ be the alternating group of 4 elements and $S=\{(123),(132),(134),(143)\}$. The set $A=H \cup H(123)$ with $H=\langle(12)(34)\rangle$ is a 2-atom of $C a y(G, S)$ which is not a subgroup. Figure 2.

Let $G$ be a group and let $S$ be a subset of $G$ such that $1 \notin S$ and $S=S^{-1}$. We say that $S$ is aperiodic if $x S \neq S, \forall x \in G$. We write $\tilde{S}=S \cup\{1\}$. Put $\Gamma=\operatorname{Cay}(G, S)$. Clearly, for every $X \subset G$,

$$
X \tilde{S}=X \cup \Gamma(X) .
$$

We shall say that $S$ is a right $r$-coprogression if $G \backslash S=\left\{a, r a, \cdots, r^{j} a\right\}$ for some $a$. Similarly we define a left $r$-coprogression. Notice that $S$ is a right $r$-coprogression if and only if $S$ is left $a^{-1} r a$-coprogression. In particular, the notions of left and right coprogressions coincide, and we shall speak only about coprogressions.

We give now a characterization of the vosperian graphs. 


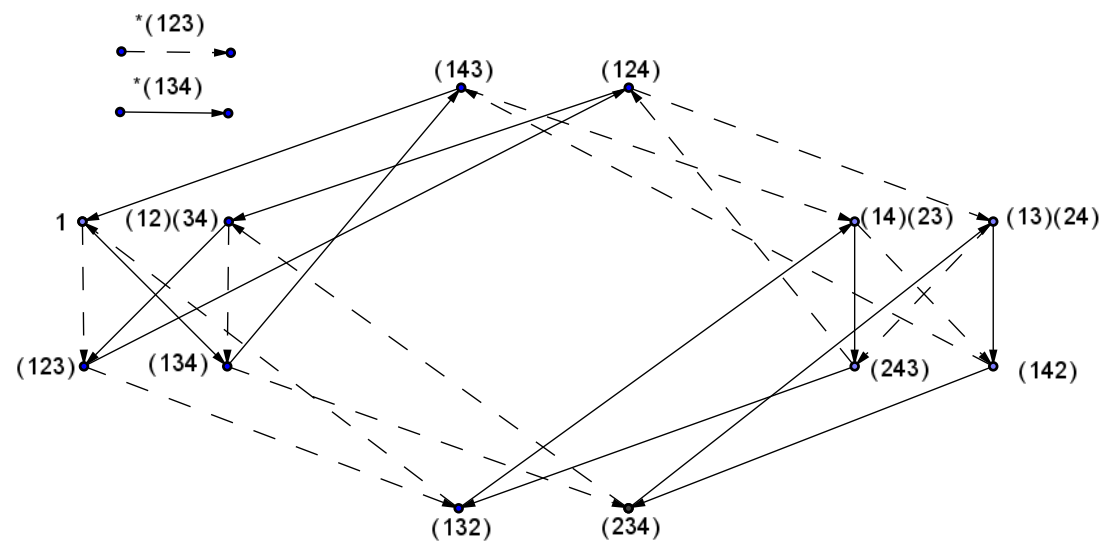

Figure 2: A graph with a 2-atom which is not a group.

Theorem 12 Let $S$ be a generating subset of a finite group $G$, with $1 \notin S, S=S^{-1}$ and $|G|-4 \geq|S|$. Then $\Gamma=\operatorname{Cay}(G, S)$ is vosperian if and only if for every $r \in G \backslash 1, \tilde{S}$ is not an $r$-coprogression, and for every subgroup $H$ and every $a \in G$ with $|H| \geq 2$,

$$
|(H \cup H a) \tilde{S}| \geq \min (|G|-1,|H \cup H a|+|S|+1) .
$$

Proof. Let us first prove the necessity. Suppose that there are a subgroup $H$ with $\mid H \geq 2$ and an element $a \in G$ such that inequality (4) does not hold. Then $\Gamma$ is 2 -separable and $\kappa_{2}(\Gamma) \leq|S|$. Suppose that there exists an $r \in G \backslash 1$, such that $\tilde{S}$ is a right $r$-coprogression. Then $|\Gamma(\{1, r\}) \cup\{1, r\}|=|\{1, r,\} \tilde{S}|=|S|+2$, and hence, $\Gamma$ is 2-separable and $\kappa_{2}(\Gamma) \leq|S|$. So each of these conditions is a necessary one.

Let us now prove the sufficiency. Suppose that $\Gamma=\operatorname{Cay}(G, S)$ is non-vosperian, that is, by Lemma E, $\Gamma$ is 2 -separable and $\kappa_{2}(\Gamma) \leq|S|$. Let $A$ be a 2 -atom of $\Gamma$ with $1 \in A$. If $\kappa_{2}(\Gamma) \leq|S|-1$, then $A$ is a 1 -atom and by Theorem C, $A$ is a subgroup. Thus inequality (4) does not hold with $H=A$ and $a=1$. So we may assume that $\kappa_{2}(\Gamma)=|S|$.

Assume first that $|A| \geq 3$. By Proposition 11, there are a subgroup $H$ and element $a \in G$ such that $A=H \cup H a$. In particular, $|H| \geq 2$ and $|G|-2 \geq|(H \cup H a) \tilde{S}|=|H \cup H a|+|S|$. So inequality (4) does not hold in this case.

Assume now that $|A|=2$, say $A=\{1, r\}$. By the definition of a 2-atom we have

$$
|\{1, r\} \tilde{S}|=|\tilde{S}|+1=|S|+2 .
$$

Let $K$ be the cyclic subgroup generated by $r$. If $G=K$, then by (5), $\tilde{S}$ is a progression and also a coprogression since $r$ generates $G$. So we may assume that $K \neq G$.

Let $\tilde{S}=S_{1} \cup \cdots \cup S_{j}$ be the trace induced on $\tilde{S}$ by a partition of $G$ into right $K$-cosets. We have $j \geq 2$ since $K \neq G$. We shall assume that $\left|S_{1}\right| \leq \cdots \leq\left|S_{j}\right|$. We must have $\left|S_{2}\right|=|K|$, since otherwise $\left|\{1, r\} S_{i}\right| \geq\left|S_{i}\right|+1$, for all $1 \leq i \leq 2$. It would follows that 
$|\{1, r\} \tilde{S}| \geq\left|S_{1}\right|+1+\left|S_{2}\right|+1+\sum_{i \geq 3}\left|S_{i}\right| \geq|S|+2$, contradicting (5). It also follows that $\left|\{1, r\} S_{1}\right|=\left|S_{1}\right|+1$. Since $r$ generates $K, S_{1}$ is a right progression with ratio $r$. Note that $K S_{1} \backslash S_{1}$ is also a right progression.

Case $1 j|K|=|G|$. In this case $G \backslash \tilde{S}=K S_{1} \backslash S_{1}$ is also a right progression.

Case $2 j|K| \leq|G|-|K|$. We must have $\left|S_{1}\right|=1$, since otherwise $(j-1)|K|+\left|S_{1}\right|-1=$ $|\tilde{S}|-1=\kappa_{2}(\tilde{S}) \leq|K \tilde{S}|-|K|=(j-1)|K|$. Thus $\left|S_{1}\right|=1$. In particular, $K$ is clearly a 2 -fragment and inequality (4) does not hold with $H=K$ and $a=1$.

Corollary 13 Let $S$ be an aperiodic generating subset of a finite group $G$, with $1 \notin S, S=S^{-1}$, and $|G|-4 \geq|S|$. Then $\operatorname{Cay}(G, S)$ is superconnected if and only if $\tilde{S}$ is not an $r$-coprogression, and for every subgroup $H$ and every $a \in G$ with $|H| \geq 2$,

$$
|(H \cup H a) \tilde{S}| \geq \min (|G|-1,|H \cup H a|+|S|+1) .
$$

Proof. One may easily see that $\Gamma=\operatorname{Cay}(G, S)$ has no twin pair since $S$ is aperiodic. By Theorem 9, $\operatorname{Cay}(G, S)$ is vosperian if and only if it is superconnected. The result follows by Theorem 12.

Let $Q=\{x: x S=S\}$. As we mentioned in the Introduction, $\bar{\Gamma}$ is the Sabidussi quotient $\mathcal{S}(G, Q, S)$. The Sabidussi quotient is a Cayley graph if $H$ is a normal subgroup. In general, it is not a Cayley graph.

Ackowlegements Research supported by the Ministry of Science and Innovation, Spain under project MTM2008-06620-C03-01/MTM. Research done when the last author was visiting Université Pierre et Marie Curie, E. Combinatoire, Paris, supported by the Ministry of Science and Innovation, Spain under the National Mobility Programme of Human Resources, Spanish National Programme I-D-I 2008-2011.

\section{References}

[1] C. Balbuena, Extraconnectivity of s-geodetic digraphs and graphs. Discrete Math. 195 (1999), no. 1-3, 39-52.

[2] C. Balbuena and A. Carmona, On the connectivity and superconnectivity of bipartite digraphs and graphs. Ars Combin. 61 (2001), 3-21.

[3] M. C. Balbuena, A. Carmona, J. Fàbrega and M. A. Fiol, On the order and size of sgeodetic digraphs with given connectivity. Discrete Math. 174 (1997), no. 1-3, 19-27.

[4] C. Balbuena, A. Carmona, J. Fàbrega and M. A. Fiol, Extraconnectivity of graphs with large minimum degree and girth. Discrete Math. 167/168 (1997), 85-100.

[5] C. Balbuena, A. Carmona, J. Fàbrega and M. A. Fiol, Superconnectivity of bipartite digraphs and graphs. Discrete Math. 197/198 (1999), 61-75. 
[6] C. Balbuena, M. Cera, A. Diánez, P. García-Vázquez and X. Marcote, Diameter-girth sufficient conditions for optimal extraconnectivity in graphs, Discrete Math. 308 (16) (2008), 3526-3536.

[7] C. Balbuena, K. Marshall and L. P. Montejano, On the connectivity and superconnected graphs with small diameter, Discrete Appl.Math. 58 (5) (2010), 397-403.

[8] F. T. Boesch, Synthesis of reliable networks. A survey, IEEE Trans. Reliab. 35 (1986) 240-246.

[9] F. T. Boesch, R. Tindell, Circulants and their connectivities, J. Graph Theory 8 (4) (1984) 487-499.

[10] J. A. Bondy and U. S. R. Murty, Graph Theory, Grad. Texts in Math. 244, Springer, 2008.

[11] B. Bollobás, Modern Graph Theory. Grad. Texts in Math. 184, Springer, 1998.

[12] J. Fàbrega and M. A. Fiol, Extraconnectivity of graphs with large girth, Discrete Math. 127 (1994) 163-170.

[13] J. Fàbrega and M. A. Fiol, On the extraconnectivity of graphs. Discrete Math. 155 (1996), no. 1-3, 49-57.

[14] M. A. Fiol, Connectivity and superconnectivity of large graphs and digraphs. Ars Combin. 29 B (1990), 5-16.

[15] M. A. Fiol, The superconnectivity of large digraphs and graphs. Discrete Math. 124 (1994), no. $1-3,67-78$.

[16] M. A. Fiol, J. Fàbrega and M. Escudero, Short paths and connectivity in graphs and digraphs, Ars Combin. 29 B (1990) 17-31.

[17] Y. O. Hamidoune, Sur les atomes d'un graphe orienté, C.R. Acad. Sc. Paris A 284 (1977), $1253-1256$.

[18] Y. O. Hamidoune, On the connectivity of Cayley digraphs, Europ. J. Combin., 5 (1984), 309-312.

[19] Y. O. Hamidoune, An isoperimetric method in additive theory. J. Algebra 179 (1996), no. $2,622-630$.

[20] Y. O. Hamidoune, Subsets with small sums in Abelian groups I: The Vosper property. European J. Combin. 18 (1997), no. 5, 541-556.

[21] Y. O. Hamidoune, Some results in Additive number Theory I: The critical pair Theory, Acta Arith. 96, no. 2(2000), 97-119.

[22] Y. O. Hamidoune, Hyper-atoms and the Kemperman's critical pair Theory, Preprint, September 2007.

[23] Y. O. Hamidoune, Some additive applications of the isoperimetric approach, Annals Insitute Fourier, Grenoble 58, 6, 2007-2008 (2008).

[24] Y. O. Hamidoune, A. Lladó and O. Serra, Vosperian and superconnected Abelian Cayley digraphs, Graphs and Combinatorics 7, 143-152 (1991). 
[25] Y. O. Hamidoune, A. Lladó, O. Serra and R. Tindell, On isoperimetric connectivity in vertex-transitive graphs. SIAM J. Discrete Math. 13, no. 1, (2000), 139-144

[26] J. van den Heuvel and B. Jackson, On the edge connectivity, hamiltonicity andd toughness of vertex-transitive graphs, J.Comb. Theory Ser. B 77, no. 1, (1999), 138-149.

[27] X. Liang, J. Meng and Z. Zhang, Super-connectivity and hyperconnectivity of vertextransitive bipartite graphs, Graphs and Combinatorics 23 (2007), 309-314.

[28] W. Mader, Über den Zusammenhang symmetricher Graphen, Arch. Math. 21 (1970), 331336.

[29] W. Mader, Eine Eigenschaft der Atome endlicher Graphen, Arch. Math. 22 (1971), 333-336.

[30] X. Marcote, C. Balbuena and I. Pelayo, Diameter, short paths and superconnectivity in digraphs. Discrete Math. 288, no. 1-3 (2004), 113-123.

[31] J. Meng, Connectivity of vertex and edge transitive graphs, Discrete Appl. Math. 127 (2003), 601-613.

[32] J. Meng and Z. Zhang, Super-connected arc-transitive digraphs, Discrete Appl. Math. 157 (2009) 653-658).

[33] G. Sabidussi, Vertex-transitive graphs. Monash. Math. 68 (1964) 426-438.

[34] O. Serra, An isoperimetric method for the small sumset problem. Surveys in combinatorics 2005, 119-152, London Math. Soc. Lecture Note Ser., 327, Cambridge Univ. Press, Cambridge, 2005.

[35] Y. Tian and J. Meng, The connectivity of vertex-transitive graphs with small regular degrees, J. Xinjiang University (Natural Science Edition), 17 (4) (2000).

[36] Y. Tian and J. Meng, Superconnected and hyperconnected 6-regular transitive graphs, J. Xinjiang University (Natural Science Edition), 25 (3) (2008).

[37] D. Wang and J. Meng, Superconnected and hyperconnected cubic transitive graphs, Or Transactions, 5 (4) (2001), 35-40.

[38] M. Watkins, Connectivity of transitive graphs, J. Combin. Theory 8 (1970) 23-29. 